University of Wollongong

Research Online

Faculty of Engineering and Information

Faculty of Engineering and Information

Sciences - Papers: Part A

Sciences

$1-1-2013$

Inertial focusing in a straight channel with asymmetrical expansioncontraction cavity arrays using two secondary flows

Jun Zhang

University of Wollongong, jz218@uowmail.edu.au

M Li

University of Wollongong

Weihua Li

University of Wollongong, weihuali@uow.edu.au

Gursel Alici

University of Wollongong, gursel@uow.edu.au

Follow this and additional works at: https://ro.uow.edu.au/eispapers

Part of the Engineering Commons, and the Science and Technology Studies Commons

Research Online is the open access institutional repository for the University of Wollongong. For further information contact the UOW Library: research-pubs@uow.edu.au 


\title{
Inertial focusing in a straight channel with asymmetrical expansion-contraction cavity arrays using two secondary flows
}

\author{
Abstract \\ The focusing of particles has a variety of applications in industry and biomedicine, including wastewater \\ purification, fermentation filtration, and pathogen detection in flow cytometry, etc. In this paper a novel \\ inertial microfluidic device using two secondary flows to focus particles is presented. The geometry of \\ the proposed microfluidic channel is a simple straight channel with asymmetrically patterned triangular \\ expansion-contraction cavity arrays. Three different focusing patterns were observed under different \\ flow conditions: (1) a single focusing streak on the cavity side; (2) double focusing streaks on both sides; \\ (3) half of the particles were focused on the opposite side of the cavity, while the other particles were \\ trapped by a horizontal vortex in the cavity. The focusing performance was studied comprehensively up to \\ flow rates of $700 \mu \mathrm{l} \mathrm{min-1.} \mathrm{The} \mathrm{focusing} \mathrm{mechanism} \mathrm{was} \mathrm{investigated} \mathrm{by} \mathrm{analysing} \mathrm{the} \mathrm{balance} \mathrm{of} \mathrm{forces}$ \\ between the inertial lift forces and secondary flow drag in the cross section. The influence of particle size \\ and cavity geometry on the focusing performance was also studied. The experimental results showed \\ that more precise focusing could be obtained with large particles, some of which even showed a single- \\ particle focusing streak in the horizontal plane. Meanwhile, the focusing patterns and their working \\ conditions could be adjusted by the geometry of the cavity. This novel inertial microfluidic device could \\ offer a continuous, sheathless, and high-throughput performance, which can be potentially applied to \\ high-speed flow cytometry or the extraction of blood cells.
}

\section{Keywords}

inertial, channel, focusing, asymmetrical, expansion, contraction, cavity, arrays, two, secondary, flows, straight

Disciplines

Engineering | Science and Technology Studies

\section{Publication Details}

Zhang, J., Li, M., Li, W. H. \& Alici, G. (2013). Inertial focusing in a straight channel with asymmetrical expansion-contraction cavity arrays using two secondary flows. Journal of Micromechanics and Microengineering, 23 (8), 1-13. 


\title{
Inertial focusing in a straight channel with asymmetrical expansion-contraction cavity arrays employing two secondary
}

\section{flows}

\section{J Zhang, M Li, W H Li* and G Alici}

School of Mechanical, Materials and Mechatronic Engineering, University of Wollongong, Wollongong, NSW 2522, Australia

E-mail:weihuali@uow.edu.au

\begin{abstract}
Focusing of particles has a variety of applications in industry and biomedicine, including wastewater purification, fermentation filtration and pathogen detection in flow cytometry, etc. Hereby, a novel inertial microfluidic device employing two secondary flows to focus particles was presented. The geometry of the proposed microfluidic channel is a simple straight channel asymmetrically patterned with triangular expansioncontraction cavity arrays. Three different focusing patterns have been observed under different flow conditions: (1) single focusing streak on cavity side; (2) double focusing streaks on both sides; (3) half of particles focused on the opposite side of cavity, and other particles trapped by horizontal vortex in cavity. The focusing performance was studied comprehensively up to the flow rates of $700 \mu \mathrm{l} \mathrm{min}^{-1}$. The focusing mechanism was investigated by analyzing force balance between inertial lift forces and secondary flow drag in the cross section. The influence of particle size and cavity geometry on the focusing performance was also studied. Experimental results showed that a more pronounced focusing performance can be obtained with large particles, which even showed a single-particle focusing streak in horizontal plane. Meanwhile, focusing patterns and their working conditions were adjustable by the geometry of cavity. The novel inertial microfluidic device was capable of offering a continuous, sheathless, and high-throughput performance, which can be potentially applied to highspeed flow cytometry or extraction of blood cells.
\end{abstract}

\section{Introduction}

Microfluidic technology has achieved a significant progress in the last decade, especially in the field of clinical diagnostics and biology. As one kind of manipulation, focusing of bio-particles, such as cells and pathogens, is essential in a lot of applications in industry and biomedicine. Focusing original 
random distributed particles into one or several equilibrium positions can be used to enrich or separate particles according to their different equilibrium positions [1]. Also, focusing of particles along a specific path can be employed in flow cytometry for detection and enumeration of bio-particles [2-3].

Various manipulation techniques have already been proposed and developed to focus particles in microfluidics, such as dielectrophoresis [4-5], magnetophoresis [6-7] and acoustophoresis [8-9]. Generally, these active methods provide a precise control of target bio-particles, but with drawbacks such as their low throughput and needing expensive equipments to provide external forces. Thus, a simple and effective passive focusing method with a high throughput is more desirable, which will significantly reduce time and cost for sample treatment. Focusing method based on particles' hydrodynamic properties meets such a demand. Inertial microfluidics, which utilizes particles' inertial migration and other effects (such as Dean flow) to manipulate particles [10], has attracted a considerable attention of microfluidics community because it is capable of treating particles sample at a very high throughput in a very simple way $[1,11]$.

The inertial migration is a phenomenon that randomly dispersed particles in the entrance of a straight channel migrate laterally to several cross-sectional equilibrium positions after a long enough distance, as shown in figure 1(a), which was observed more than 50 years ago [12-15]. The most remarkable observation was conducted by Segre and Silberberg [15-16]. In their experiments, macroscopic neutrally buoyant spheres suspended in Poiseuille flow migrated laterally to a narrow annulus at about 0.6 times of tube radii from the axis. This was a significant challenge to the widelyaccepted concept that neutrally suspended particles would follow the fluid streamlines. Afterwards, although a number of experimental studies and theoretical analyses were conducted to explore the mechanism of this migration phenomenon [12, 17-21], it had not been taken into account in the practical application until the raise of microfluidic technology recently, where particles' size is comparable with characteristic dimension of microchannel. Specifically, this inertial migration phenomenon was used to focus and filter particles [22-26]. Mach and Di Carlo [23] presented a series connected straight channel system to filter pathogenic bacteria with an efficiency more than $80 \%$. Hou et al. [26] demonstrated to separate Escherichia coli and Saccharomyces cerevisiae from whole blood at a removal efficiencies of about $80 \%$ and $90 \%$ respectively in a narrow $(20 \times 20 \mu \mathrm{m})$ straight channel.

Furthermore, curved channels can create an additional secondary flow effect to assist and modify particles' inertial migration process. The secondary flow is induced by a pressure gradient in radial direction, because of fluid velocity mismatching in the centre and near-wall region in the downstream of a curved channel [10]. Compared to straight channel, curved channel has following superior advantages: (i) improvement of collection purity due to the adjustment of particles equilibrium positions; (ii) reduction of channel footprint for particles' lateral migration due to the assistance of secondary flow to accelerate lateral migration; and (iii) equilibrium separations of particles based on different equilibrium positions of particles with various sizes [10]. The reported curving geometry for 
the inertial microfluidics includes spirals [27-31], single arc [32-34] and symmetric and asymmetric serpentine [1, 11, 33, 35]. Bhagat et al. [31] demonstrated a complete separation of $7.32 \mu \mathrm{m}$ and 1.9 $\mu \mathrm{m}$ particles in a 5-loop spiral microchannel. Vermes et al. [30] used a planar spiral microchannel to spatially order two types of myeloid leukemic cells (HL60 and K562 cells), enabling a 77\% efficiency of deterministic single cell encapsulation in picolitre drops. Oakey et al. [35] evaluated the performance of particles focusing in asymmetric serpentine channels using standard flow cytometry methods, and found that their devices can operate with increasing effectiveness at higher flow rates and particle concentrations.

Besides the curved channel, it was also reported that secondary flow, existing in a straight channel with expansion-contraction cavity arrays (CEA channel), can also be employed to modify inertial migration of particles [36-38]. Two kinds of secondary flows can be created in CEA microchannel including circulating vortex in horizontal plane, due to the detachment of fluid boundary layer in the channel expansion area at a large inertia [38-40], and counter-rotating flow in cross-sectional plane [36], produced by radial pressure gradient in the downstream of cavity contraction area, similar as the Dean flow in curved channel. Circulating vortex in horizontal plane was investigated extensively to trap specific bio-particles (such as CTCs) from mainstream according to their sizes [39-40], as shown in figure 1(b). Furthermore, bio-particles were circulated in horizontal vortex to study the effects of centrifugal force and shear stress on their morphology [41-43]. The secondary flow in cross-sectional plane has been applied to focus [44-45] and separate particles [3637]. Park et al. [44] presented a multi-orifice microfluidic channel to focus microparticles by the combination of inertial migration and secondary flow in cross-sectional plane. In their device, the cavity was rectangular and symmetrically patterned on two sides of a straight channel. Inspired from the idea that asymmetry can improve the sorting efficiency in spiral channel [11], Lee et al. [36-37, 45] developed a microfluidic device with asymmetrically patterned rectangular cavity arrays to achieve a three dimensional focusing and extraction of blood cells from blood plasma with a $62.2 \%$ yield and $1.2 \mathrm{ml} \mathrm{h}^{-1}$ throughput. However, a sheath flow was needed while their device operating, which brought potential drawbacks of dilution and contamination on bio-particles sample, as well as complication of the whole microfluidic system.

To the authors' knowledge, inertial microfluidic chip employing both of two secondary flows together to focus or sort particles has not yet been explored. In order to extend the applications of these secondary flows in microfluidics, we propose a novel inertial microfluidic device, employing two secondary flows in cross-sectional and horizontal planes, to achieve three different focusing patterns in a CEA microchannel. Besides, no sheath flow is needed in our microfluidic device, and this eliminates the risk of contamination and dilution. The proposed microfluidic device can provide a continuous, sheathless and high-throughput focusing performance, which has a variety of potential applications. 


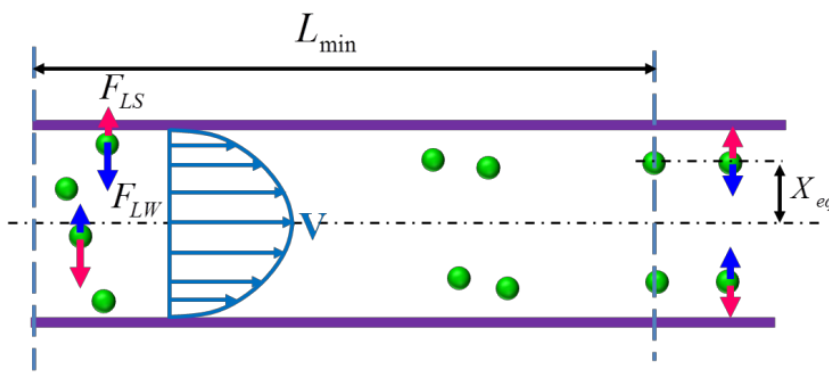

(a)

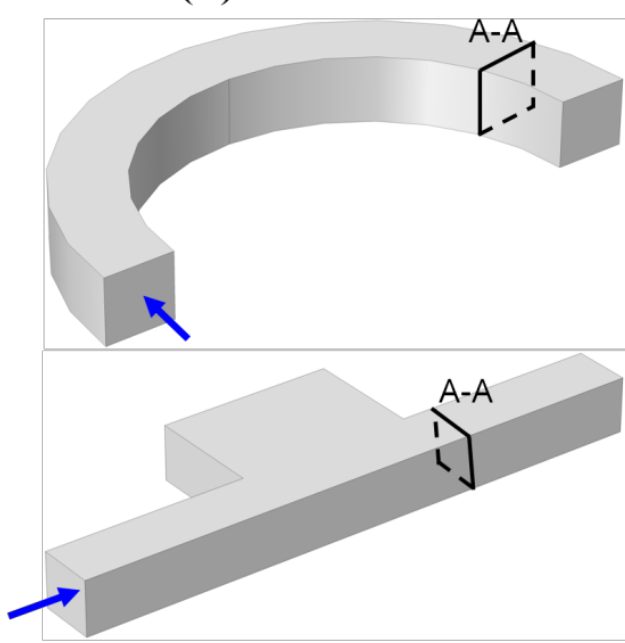

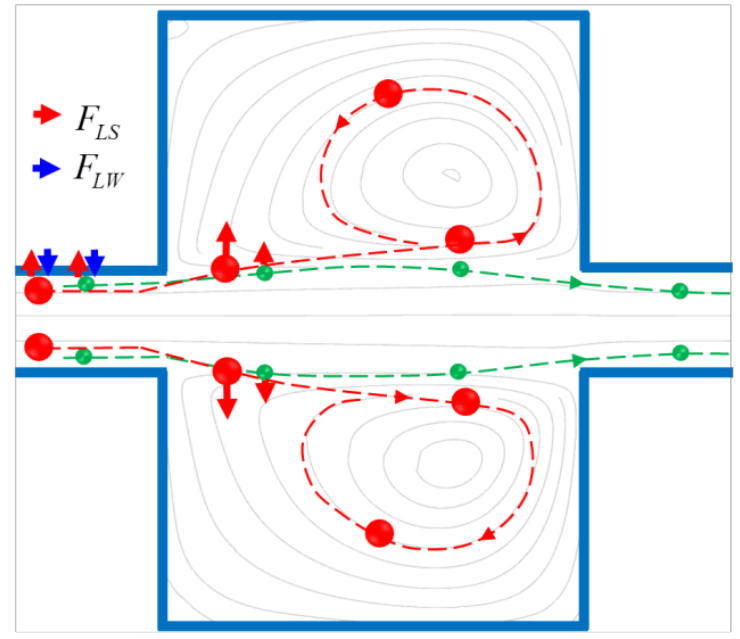

(b)

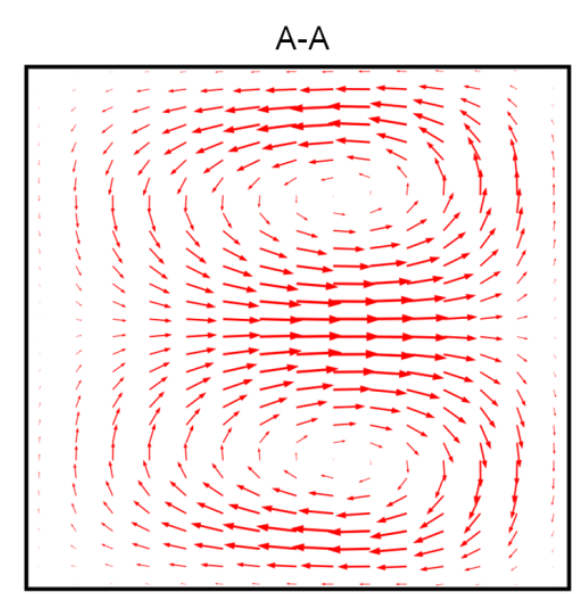

(c)

Figure 1. (a) Neutrally buoyant particles migrate laterally in a straight channel due to non-zero net inertial lift force, and reach their equilibrium positions after an enough distance. (b) A horizontal vortex, caused by the detachment of fluid boundary layer at high flow rate, can be applied to selectively trap particles from main stream according to particles' size [39]. (c) Two counter-rotating vortices in the cross-section of curved channel [10] and CEA channel [37].

\section{Theoretical backgrounds}

\subsection{Particle focusing in a straight channel}

The inertial migration of particles in a straight channel is driven by the counteraction of two inertial effects: shear gradient lift force $F_{L S}$, and wall lift force $F_{L W}$. The shear gradient lift force $F_{L S}$ is due to the curvature of fluid velocity profile, while the wall lift force $F_{L W}$ is a result of wall-induced disturbance on the flow field around the suspended particles. The existence of equilibrium positions is resulted from the balance of two inertial lift forces. The expressions for these two lift forces are hard to obtain as they are always complexly coupled. But their net inertial lift force was first derived by Asmolov based on the method of matched asymptotic expansions [19], and later simplified by Di Carlo [10] as following: 


$$
\begin{gathered}
F_{L}=\frac{\rho_{f} U_{m}^{2} a^{4}}{D_{h}^{2}} f_{L}\left(R_{C}, x_{C}\right) \\
R_{C}=\frac{\rho_{f} U_{m} D_{h}}{\mu_{f}}
\end{gathered}
$$

where $\rho_{f}, U_{m}$ and $\mu_{f}$ are fluid density, maximum velocity and dynamic viscosity, respectively. $a$ is spherical particles' diameter. $D_{h}$ is hydraulic diameter of channel, defined as $D_{h}=D$ for a circular channel ( $D$ is the diameter of circular cross-section) or $D_{h}=2 w h /(w+h)$ for a rectangular channel ( $w$ and $h$ correspond to width and height of the rectangular cross-section). $f_{L}\left(R_{C}, x_{C}\right)$ is lift coefficient of net inertial lift force, and it is a function of particles position within cross-section of channel $x_{C}$ and channel Reynolds number $R_{C}$ [10]. The Reynolds number $R_{e}$ based on the fluid average velocity is related with $R_{C}$ as: $R_{e}=2 R_{C} 3$. The situation where $f_{L}=0$ corresponds to the equilibrium positions of particles. Although $f_{L}$ varies with Reynolds number, at $R_{C}<100$ that is typical for most microfluidic applications, the lift coefficient remains relatively constant, and can be approximated averagely as $f_{L} \approx 0.5$ [11]. In straight channel, particles’ lateral migration velocity $\left(U_{L}\right)$ and the minimum channel length $\left(L_{\min }\right)$, as shown in figure 1(a), which is required for particles to migrate to their equilibrium positions, can be derived by balancing the net inertial lift force and stokes drag [46]:

$$
\begin{gathered}
F_{\text {stks }}=3 \pi \mu_{f} a U_{L} \\
U_{L}=\frac{F_{L}}{3 \pi \mu_{f} a}=\frac{\rho_{f} U_{m}^{2} a^{3}}{6 \pi \mu_{f} D_{h}^{2}} \\
L_{\text {min }} \approx \frac{D_{h}}{2 U_{L}} * U_{m}=\frac{3 \pi \mu_{f} D_{h}^{3}}{\rho_{f} U_{m} a^{3}}
\end{gathered}
$$

Two dimensionless Reynolds numbers can characterize the lateral migration of particles in straight channel, which are channel Reynolds number $R_{C}$ describing the ratio between inertial force and viscous force of fluid in a flow, and particle Reynolds number $R_{P}$ additionally considering the ratio of particle size to channel size.

$$
R_{P}=R_{C} \frac{a^{2}}{D_{h}^{2}}=\frac{\rho_{f} U_{m} a^{2}}{\mu_{f} D_{h}}
$$

When $R_{P}<<1$, the viscous interaction of fluid and particles dominates the particles' movement in the channel. Under this condition, particles are subjected to the dominant surface drag to follow fluid streamlines. However, increasing $R_{P}$ to the order of 1 , inertial lift forces become dominant that lateral migration of particles across the fluid streamlines becomes obvious [11].

\subsection{Effects of Secondary flow}

\subsubsection{Secondary flow in the cross-sectional plane}


When channels are not single straight but curved or with expansion-contraction cavity (or CEA channel), an additional secondary flow in cross-sectional plane appears, as shown in figure 1(c). Secondary rotational flow, also called Dean flow, caused by the radial pressure gradient in curved channel, was first reported to enhance the species mixing in microfluidics [47]. Recently, it was applied to modify particles' trajectory in inertial focusing processes [1, 31, 33, 35]. Two dimensionless numbers were used to characterize this secondary flow: Dean number $D e=R_{e}(H / 2 R)^{1 / 2}$ and curvature ratio $\delta=H / 2 R$, where $R$ is the radius of channel curvature, and $H$ is width of channel. The secondary flow velocity $\left(\mathrm{U}_{\mathrm{D}}\right)$ is scaled as: $U_{D} \sim D e^{2}[10]$.

Meanwhile, rotational flow can also happen in a straight channel with expansion-contraction cavity arrays (CEA channel) due to the abrupt change of cross-sectional area. The acceleration of fluid in the contraction area can produce a Dean-like counter-rotating flow (vortex). Recently, this secondary flow effect was already explored to applications of particle focusing and separation [37, 45, 48]. The secondary flow drag $F_{D}$, which is due to the difference between particle velocity and fluid velocity in cross-sectional plane, can be calculated by stokes drag law:

$$
F_{D}=3 \pi \mu_{f} a\left(v_{f}-v_{P}\right)
$$

where $v_{f}$ and $v_{p}$ are the lateral velocities of fluid elements and particles in cross-sectional plane, respectively. In this paper, the symbol for Dean drag in curved channel and Dean-like (secondary flow) drag in the CEA channel were not distinguished as they both could be summarised as secondary flow drag $F_{D}$.

In curved channel, Dean drag $F_{D}$ co-works with inertial lift forces $F_{L}$ on particles in crosssectional plane. When $F_{D}$ is of the same order as $F_{L}$, equilibrium positions due to the inertial effects can be modified to new focusing positions. If $F_{D}>>F_{L}$, no focusing position is observed, as Dean drag $F_{D}$ is dominant to mix particles in the rotating flow. If $F_{D}<<F_{L}$, the focusing induced by inertial lift forces alone is achieved in the downstream [11].

\subsubsection{Secondary flow in horizontal plane: horizontal vortex in a CEA channel}

At a high fluid inertia, horizontal vortex in the expansion area of a channel is induced due to the detachment of fluid boundary layer. This horizontal vortex works with inertial migration to selectively trap particles according to their sizes [39-40]. When focused particles, which are the results of inertial migration after a long enough distance in a straight channel, reach expansion region, the neighbouring channel wall is no longer within its vicinity. It leads to the sudden loss of wall lift force $F_{L W}$ to balance the remaining shear gradient lift force $F_{L S}$ which directs to the vortex. Then particles transfer laterally to the vortex, and their migration velocity $v_{t}$ is:

$$
v_{t}=F_{L S} / 3 \pi \mu_{f} a=\rho_{f} U_{m}^{2} a^{2} f_{L S}\left(R_{C}, x_{C}\right) / 3 \pi \mu_{f} D_{h}
$$

where $f_{L S}\left(R_{C}, X_{C}\right)$ is the lift coefficient of shear gradient lift force which depends on the local shape of fluid velocity profile and channel Reynolds number [40, 49]. It indicates that particles larger than a critical size are expected to migrate across streamlines into vortex, whereas smaller particles cannot 
migrate fast enough into the vortex before passing the expansion-contraction cavity area. In this way, particles can be selectively trapped and separated according to their sizes [40].

\section{Materials and methods}

\subsection{Design and fabrication of microfluidic device}

The isosceles right-angled triangular cavities were patterned on one side of a straight channel. The aspect ratio of the straight channel was 1.4, with a cross-section of $50 \mu \mathrm{m} \times 70 \mu \mathrm{m}$ (width $\times$ height). The maximum edge of the triangle was $L_{1}=900 \mu \mathrm{m}$, and the space between two adjacent cavities was uniform as $L_{2}=900 \mu \mathrm{m}$. The total length of straight channel was $L_{0}=31 \mathrm{~mm}$, including 17 repeated expansion-contraction triangular cavities. Its schematic geometry is shown in figure 2.

The device was fabricated by standard photolithography and soft lithography techniques. The fabrication included three main steps: rapid prototyping of a silicon master, Polydimethylsiloxane (PDMS) replica moulding, and sealing through plasma oxidation. Briefly, photoresist (SU-8 2025, MicroChem Corp., Newton, MA) was spun at $2000 \mathrm{rpm}$ to a thickness of $70 \mu \mathrm{m}$ on a silicon wafer, and then exposed to UV light through a designed mask using a mask aligner system (ABM, San Jose, CA). After that, the photoresist on the silicon wafer was developed in a SU-8 developer solution and rinsed by isopropylalcohol (IPA) to create a positive replica of channel geometry. PDMS mixture with a 10:1 ratio of base to agent (Dow Corning, Midland, MI) was poured over the silicon master, degassed to remove bubbles in a vacuum oven, and cured at $100^{\circ} \mathrm{C}$ for 45 mins. When PDMS was cured, it was taken out of silicon master, and inlet and outlet holes were punched with a custom needle tip. Finally, PDMS slide was bonded with a clean glass slide after exposure to oxygen plasma (PDC-002, Harrick Plasma, Ossining, NY) for 3 mins.

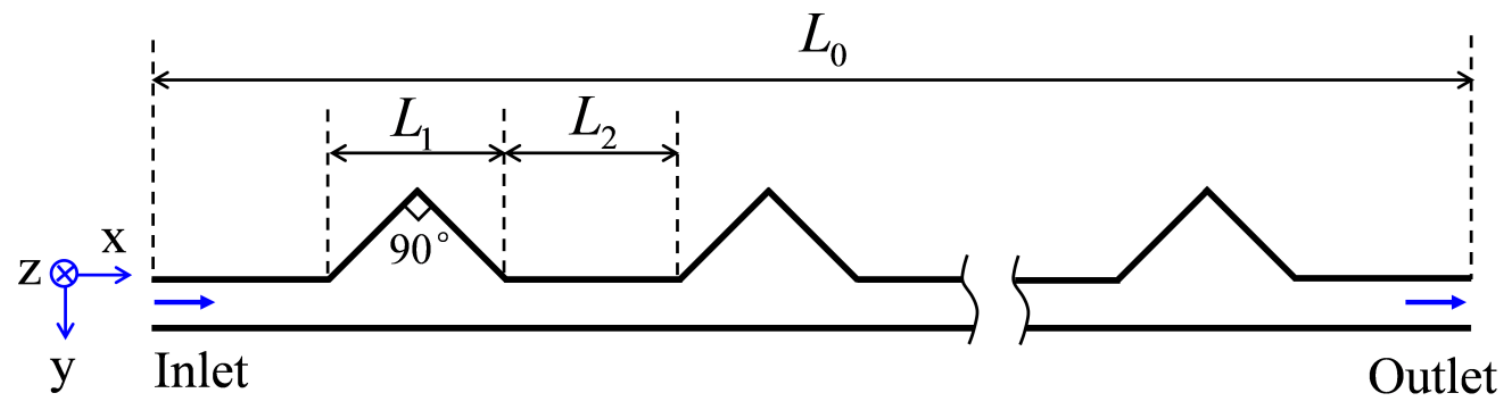

Figure 2. The schematic geometry of the microfluidic channel with triangular expansion-contraction cavity arrays, $L_{0}=31 \mathrm{~mm}, L_{1}=900 \mu \mathrm{m}, L_{2}=900 \mu \mathrm{m}$.

\subsection{Particles suspension}

Internally dyed fluorescent polystyrene microspheres were purchased from Thermo Fisher Scientific., USA. Particles suspension was prepared by diluting $9.9 \mu \mathrm{m}$ (Product No.G1000, CV $<5 \%$ ), $4.8 \mu \mathrm{m}$ (Product No.G0500, CV<5\%) and $3.2 \mu \mathrm{m}$ (Product No.G0300, CV<5\%) particles suspension to the 
concentration of $\sim 10^{5}$ particles $\mathrm{ml}^{-1}$ by deionized water. This concentration was considered as low enough to neglect interaction of particles in micro-channel.

\subsection{Experimental setup}

Particles suspensions were shaken vigorously before being transferred to a $5 \mathrm{ml}$ syringe, and then introduced into the microfluidic chip through a silicon tube by a syringe pump (Legato 100, Kd Scientific). The outflow of particles suspension was collected in a glass bottle. The microfluidic chip was put in an inverted microscope (CKX41, Olympus, Japan), illuminated by a mercury arc lamp. The fluorescence images of fluorescent particles were observed and captured by a CCD camera (Rolera Bolt, Q-imaging, Australia) which had a maximum capturing speed of 30 frames per second. The fluorescence images were then post-processed and analyzed in the software Q-Capture Pro 7 (Qimaging, Australia). The flow rate in the experiment was increased from 50 to $700 \mu \mathrm{l} \mathrm{min}{ }^{-1}$, corresponding to the average fluid velocity from 0.238 to $3.332 \mathrm{~m} \mathrm{~s}^{-1}$. The exposure time for each frame was ranged from 100 to $200 \mathrm{~ms}$, depending on magnification and flow rate. The fluorescence intensity profile was taken from the outlet of last cavity to exam the focusing performance of this microfluidic device. Focusing width was determined by measuring the distance between points where intensity profile crossed 50\% threshold. Focusing position was taken as the middle of 50\% threshold intensity.

\subsection{Numerical simulation}

COMSOL Multiphysics 4.2 (COMSOL, Burlington, MA) finite element software was employed to calculate the flow field in a straight channel with only 2 of 17 expansion-contraction cavities to save computational time and space. The calculated flow field was used to analyze the mechanism of particles focusing in microfluidic channel. The reason of using two cavities is to check whether the disturbance of previous cavity on flow filed will affect the inlet condition of the following cavity, then influence its flow field and corresponding movement of particles within it. The laminar steady incompressible flow model was used, because the maximum Reynolds number in the experiments was about 250, far less than 2300 which was normally considered as the transition number form Laminar to turbulent flow [50]. The inlet condition was set with a uniform average velocity calculated from the flow rate, and the non-slip boundary condition was applied on channel surfaces.

\section{Results and discussion}

\subsection{Effects of flow condition}

The $9.9 \mu$ m particles suspension was introduced into the microfluidic chip with a flow rate ranging from 50 to $700 \mu \mathrm{l} \mathrm{min}^{-1}$ in the experiments. Three different focusing patterns were observed under different flow conditions: (i) single focusing streak on cavity side (figure 3(a)); (ii) double focusing streaks on both sides (figure 3(b)); and (iii) half of particles focused on the opposite side of cavity, 
and other particles trapped by vortex in cavity (figure 3(c)). The fluorescence images of $9.9 \mu \mathrm{m}$ particles and their focusing positions at the outlet are plotted in figure 4.

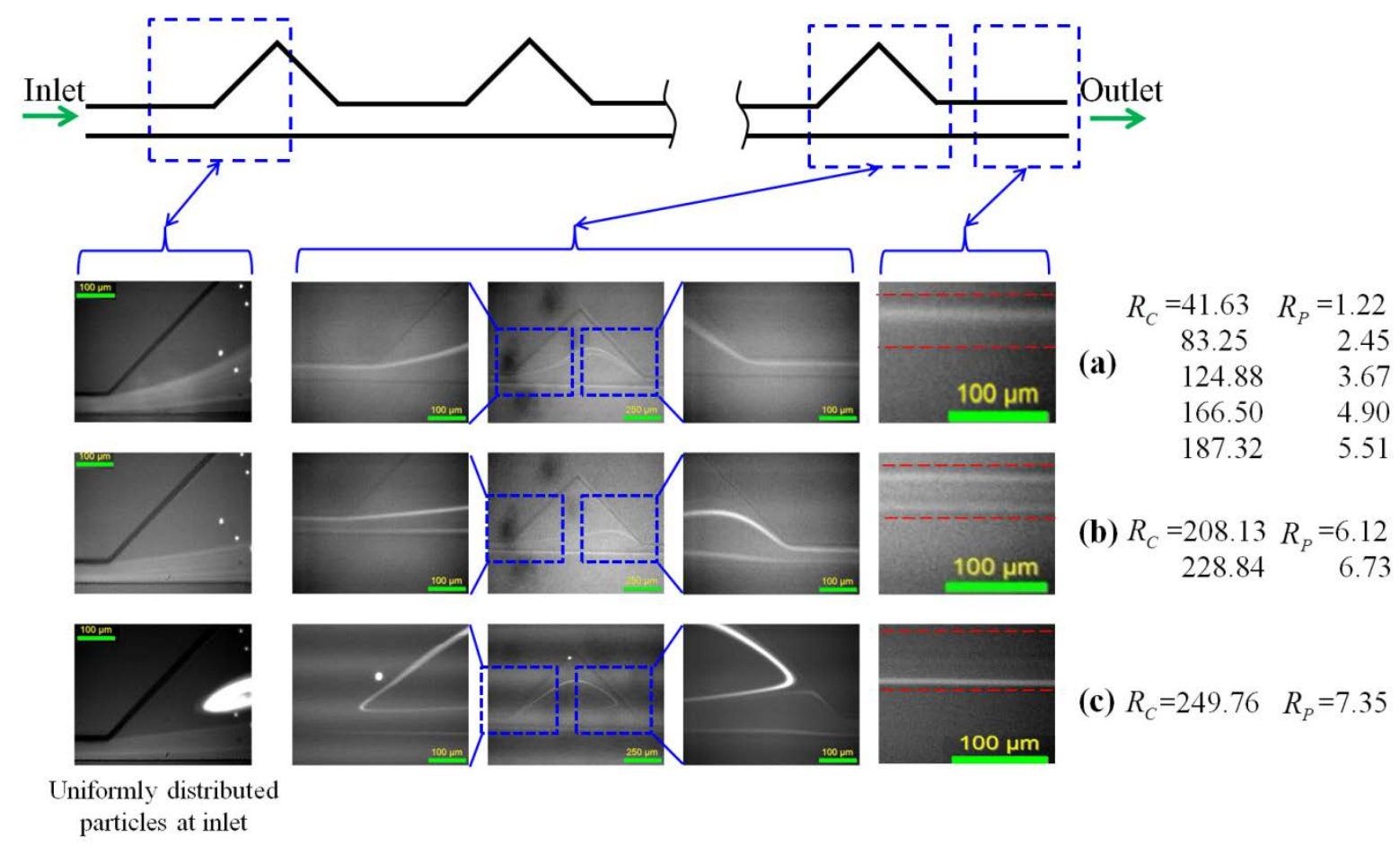

Figure 3. Three different focusing patterns were observed under different flow conditions for $9.9 \mu \mathrm{m}$ particles. (a) Single focusing streak on the cavity side. (b) Double focusing streaks on both sides. (c) Half of particles focused on the opposite side of cavity, and other particles trapped by vortex in cavity.

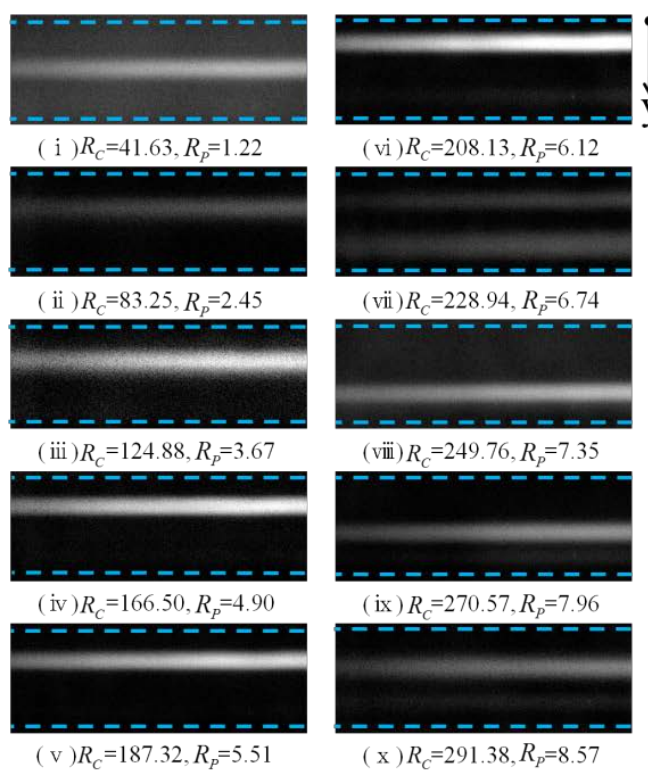

(a)

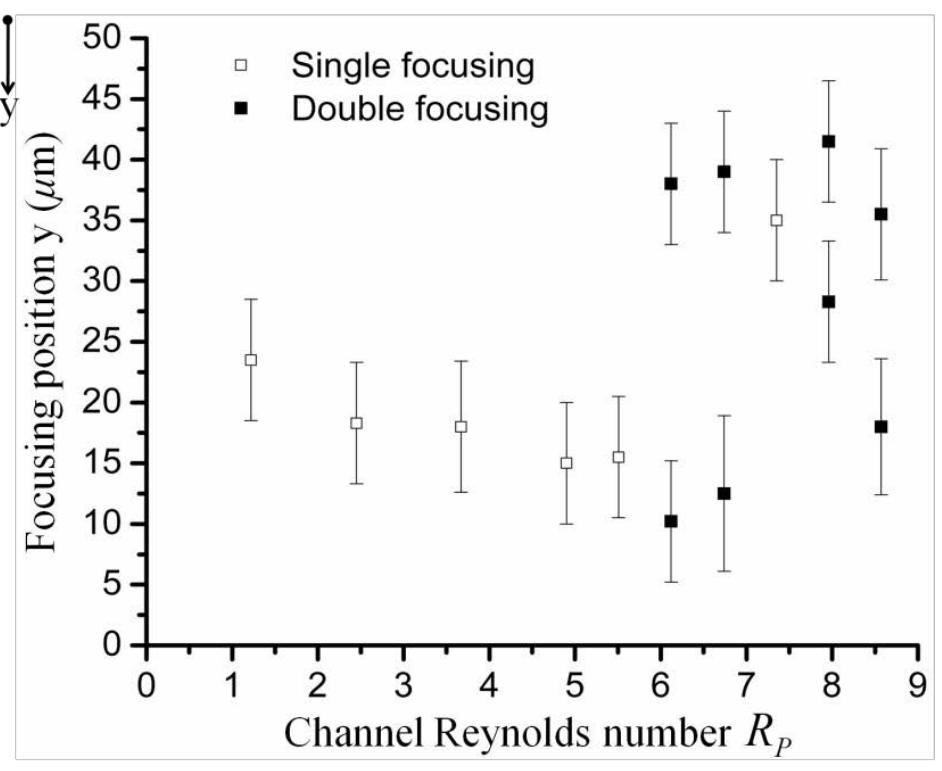

(b)

Figure 4. (a) Fluorescence images at the outlet indicate the focusing positions of $9.9 \mu \mathrm{m}$ particles. (b) The focusing positions at the outlet under different particle Reynolds numbers. Error bars are the particles focusing width. 
(i) Increase the input flow rate from $50 \mu \mathrm{l} \min ^{-1}\left(R_{C}=20.82, R_{P}=0.61\right)$ at the beginning, the focusing phenomenon was not obvious until it was up to $100 \mu \mathrm{lmin}^{-1}\left(R_{C}=41.63, R_{P}=1.22\right)$. It coincided with the results in a straight channel where inertial migration became apparent when particle Reynolds number was of order 1 [11]. Different from that at least two focusing streaks existed in the straight channel $[10,46]$, a single focusing streak was observed near the centre of channel (figure 4(b)). It can be explained by the fact that secondary flow drag $F_{D}$ superposed on the inertial effects is big enough to modify the original inertial equilibrium positions. Additionally, the mainstream velocity in the primary direction is quite slow, which allows enough time for the transport of particles to the new modified equilibrium positions. In order to verify that the magnitudes of inertial lift forces and secondary flow drag are of the same order, they are calculated and compared qualitatively. The average net inertial lift force $F_{L}$ here is about $0.83 \mathrm{nN}$, when $f_{L}=0.5$ (equation (1)). As the actual particle velocity is unknown, the exact secondary flow drag is hard to get. However, the maximum drag in the lateral direction can be obtained based on the assumption of zero lateral velocity of particles [51]. The maximum secondary flow drag $F_{D}$ is about $1.1 \mathrm{nN}$ (equation (7)), where fluid lateral velocity $v_{f}$ is calculated averagely based on the flow field in the cross section (b) (shown in figure 5(a)). So it can be concluded that inertial lift forces and secondary flow drag are qualitatively in the same order under this flow condition.

For better understanding of focusing mechanism, we conducted a numerical simulation for the flow field in a straight channel with two triangular expansion-contraction cavities when the flow rate is $100 \mu \mathrm{l} / \mathrm{min}$, as shown in figure 5 (b) $\left(R_{C}=41.63, R_{P}=1.22\right)$. Generally, an expansion-contraction cavity induces a disturbance on primary flow in the straight channel section, especially in the cross section. When the fluid accelerates in the contraction area, a Dean-like counter-rotating vortex is formed in the downstream cross section (b) (figure $5 b(i i)$ ). The influence of cavity weakens along the flow direction $x$ and becomes negligible in the middle of two cavities (cross section (c), figure $5 b(i i i))$. So the influence of previous cavity on the flow field does not reach the following cavities.

It is well known that when particles are flowing in a straight channel, they experience two inertial lift forces: shear gradient lift force $F_{L S}$ directing towards the channel walls and wall lift force $F_{L W}$ pointing to channel centreline. The balance of these two inertial lift forces leads to several equilibrium positions within the cross section. In a square straight channel (AR=height/width=1), particles focus to four equilibrium positions, at the centre of each face. Additionally, a further reduction to two equilibrium positions happens in a high aspect ratio ( $A R>1)$ channel, particles focus to the centre face about $0.2 D_{h}$ away from the side walls [37, 48, 52]. When particles are flowing in a high-aspect-ratio channel with expansion-contraction cavity arrays, an additional secondary flow drag exerts on the particles. The original two inertial equilibrium positions (net inertial lift force $F_{L}=0$ ) become unstable due to the disturbance of counter-rotating streamlines in cross section (b), and only one modified equilibrium position survives on the cavity side. As shown in figure 5c(i), particles on the right (blue circle) experience net inertial lift force $F_{L}$ and secondary flow drag $F_{D}$ which both direct 
approximately to the right walls, and a little unbalance of these forces in $\mathrm{z}$ direction will drive particles into the top or bottom rotating streamlines. For the particles on the top and bottom (aqua circles), they will easily follow the rotating streamlines due to the strong secondary flow drag, and finally reach then stay at the single modified equilibrium position (green circle) where $F_{L}$ and $F_{D}$ counteract equally to each other. The modified equilibrium position is expected much further from the left wall than the original left inertial equilibrium position (dashed circle in figure 5c(iii)), because only a wall-directed net inertial lift force $F_{L}$ can balance the centreline-directed secondary flow drag $F_{D}$. This is also proven by experimental results that particle focusing position is $23.8 \mu \mathrm{m}$ away from left wall (figure 4(b)), which is much larger than $11.7 \mu \mathrm{m}\left(0.2 D_{h}\right)$ for left inertial equilibrium position. Also, the focusing width is about $10 \mu \mathrm{m}$ (figure 6(a)), equal to the diameter of particles. It means that a single-particle focusing (all particles focus at one equilibrium position in the cross section, as depicted in figure $5 c($ iii)) is achieved and it agrees well with our expectation. Additionally, numerical modelling was employed to predict particles' trajectory in the microchannel. The inertial lift forces and secondary flow drag are believed to dominate this single-particle focusing, although centrifugal force of particles in each turn may affect the focusing process (more information see supplementary figure S1 and the corresponding text).

(a)

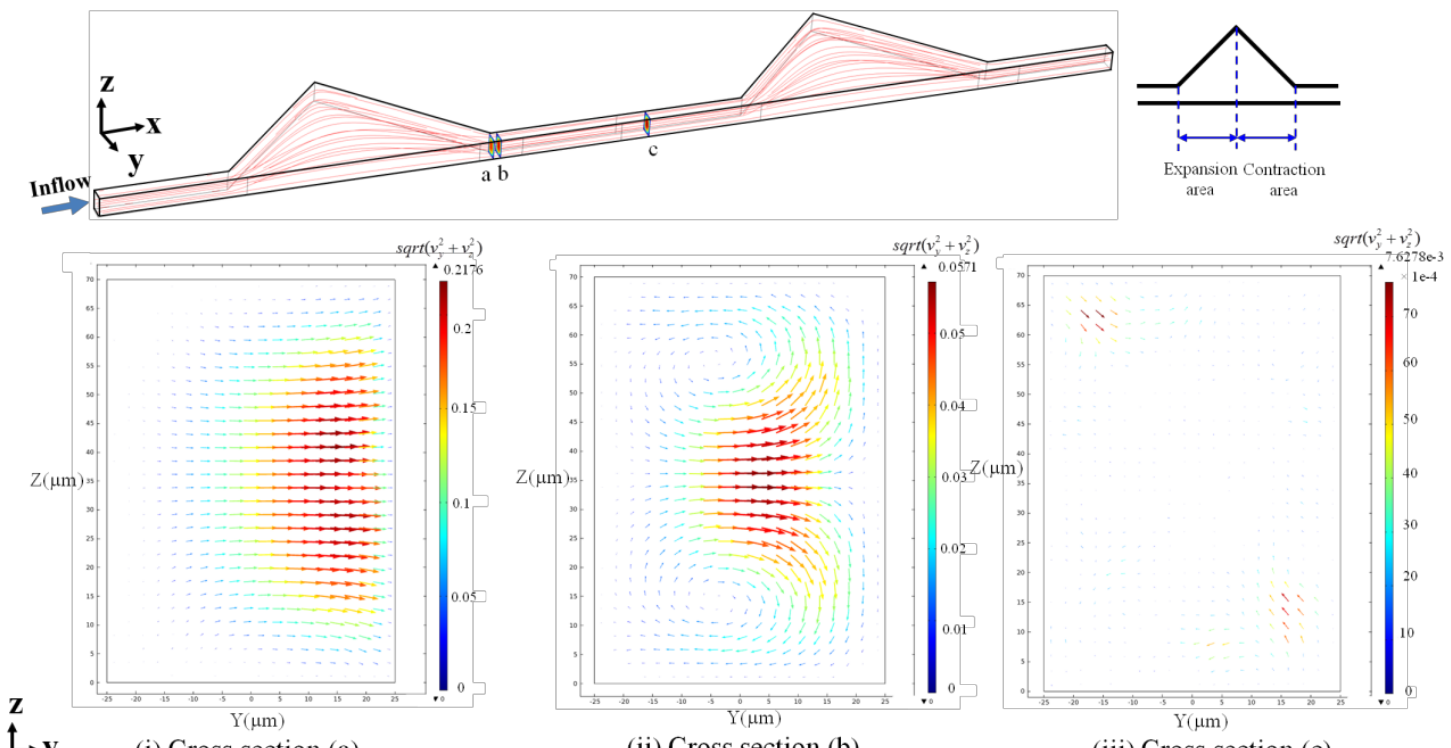

(i) Cross section (a)

(ii) Cross section (b)

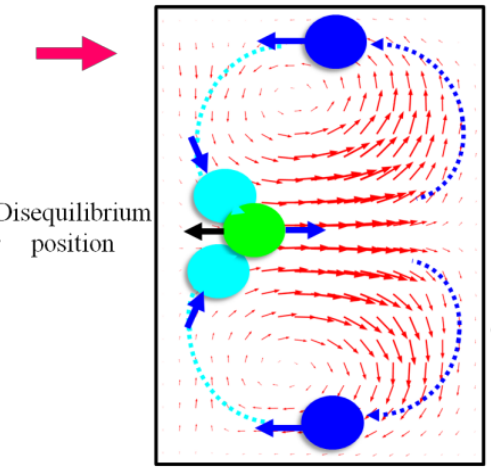

(ii) (iii) Cross section (c)

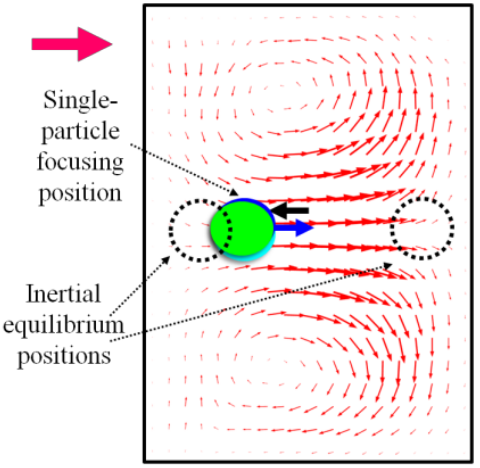

(iii) 
Figure 5. (a) A straight channel with two triangular expansion-contraction cavities. (b) The simulation results of flow field in the cross section (a) (c), which are $900 \mu \mathrm{m}, 925 \mu \mathrm{m}$ and $1350 \mu \mathrm{m}$ respectively from the left beginning of first triangular cavity along $\mathrm{x}$ direction. (c) Schematic illustration of focusing mechanism in a straight channel with expansion-contraction cavity arrays. A single equilibrium position is resulted from the balance between the net inertial lift force $F_{L}$ and secondary flow drag $F_{D}$, with its position (solid circle) closer to the centreline of channel than original left inertial equilibrium position (dashed circle).

(ii) Continue to increase flow rate, the single focusing streak shifted to cavity side, and it arrived at $y=15.5 \mu \mathrm{m}$ when flow rate was $450 \mu \mathrm{min}^{-1}\left(R_{P}=5.51\right.$, figure $\left.6(\mathrm{~b})\right)$. When the flow rate exceeded $500 \mu \mathrm{l} \mathrm{min} \operatorname{mos}^{-1}\left(R_{P}=6.12\right)$, another focusing streak on the opposite side of cavity was on set at $y=38 \mu \mathrm{m}$ (figure 6(c)). At this time, the inertial migration began to dominate the particles' movement in the cross-sectional plane, and two equilibrium positions existed which was same as the situation in a straight channel [22]. In order to verify that the inertial lift forces are superior to the secondary flow drag at this situation, both the inertial lift forces and the secondary flow drag force are scaled with input flow velocity. From figure 7 , it can be seen that the magnitude of secondary flow velocity $v_{f}$ is proportional to the input velocity: $v_{f} \sim U_{m}$. Meanwhile, the secondary flow drag $F_{D}$ is proportional to $v_{f}$ by the stokes drag law [51], therefore $F_{D} \sim U_{m}$. However, from equation (1), the net inertial lift force is proportional to the square of input velocity, $F_{L} \sim U_{m}{ }^{2}$, which means that the inertial lift forces increase much faster than the secondary flow drag when $U_{m}$ is over than $1 \mathrm{~m} \mathrm{~s}^{-1}$. So after flow rate exceeds a certain level (500 $\left.\mu \mathrm{l} \mathrm{min}^{-1}, U_{m}=3.57 \mathrm{~m} \mathrm{~s}^{-1}\right)$, the secondary flow drag becomes negligible compared to the inertial lift forces.

(iii) When the flow rate reached $600 \mu \mathrm{l} \mathrm{min}^{-1}\left(R_{P}=7.35\right)$, the fully developed vortex in the cavity area began to trap particles from the mainstream on the cavity side, and isolated them in circulating streams (figure 3(c)). So a single focusing streak on the opposite side of cavity was observed at $y=35$ $\mu \mathrm{m}$ (figure 6(c)). The trapping of particles happens due to the disappear of wall lift force which always balances the shear gradient lift force in a straight channel, so particles can migrate across streamlines into vortex [39-40]. This focusing pattern is expected to satisfy demands where the particles focusing and trapping need to be conducted simultaneously. For example, half of particles can be trapped in the vortex for the labelling and study of shear stress on the morphology of bioparticles, and the other particles are focused along a specific path for the detection and enumeration in the downstream.

The single focusing streak moved from right to left side when flow rate surpassed $650 \mu \mathrm{l} \mathrm{min}{ }^{-1}$ $\left(R_{P}=7.96\right)$, and also a new focusing streak seemed to arise around its original position (figure 6(d)), which may be due to the saturation of particles in the vortex region.

There may also be the concern whether Dean-like vortex will change or even disappear due to the development of horizontal vortex in the cavity as they are actually intrinsically linked. Hereby, the flow field under three typical flow conditions were calculated by numerical simulation: (1) no 
horizontal vortex, (2) partially developed horizontal vortex, and (3) completely developed vortex, as shown in figure 8. It was found that even the horizontal vortex was developed completely, the Deanlike vortex still stayed, but its shape became a little flat. Additionally, as mentioned above, the inertial lift forces dominated the particles' movement in the cross sectional plane before horizontal vortex was developed completely. At this point, the secondary flow drag could be neglected, and the crosssectional flow field (or Dean-like vortex) actually was not important in the second and third focusing pattern.

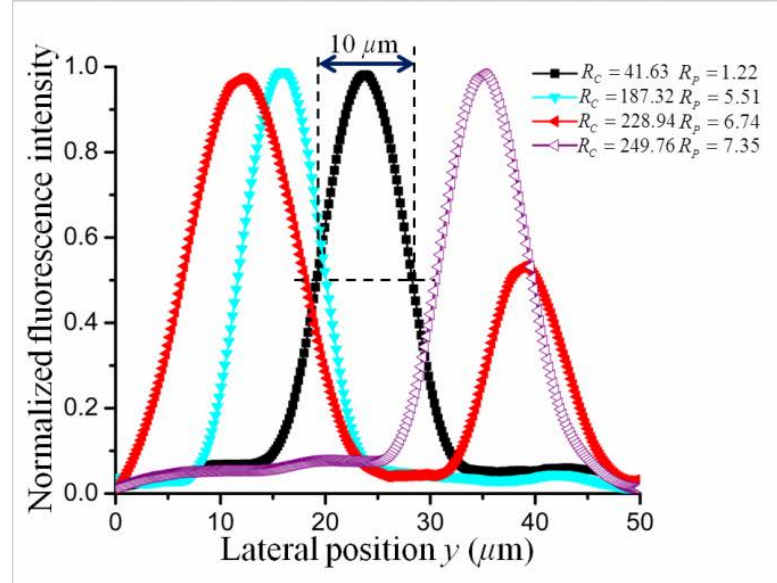

(a)

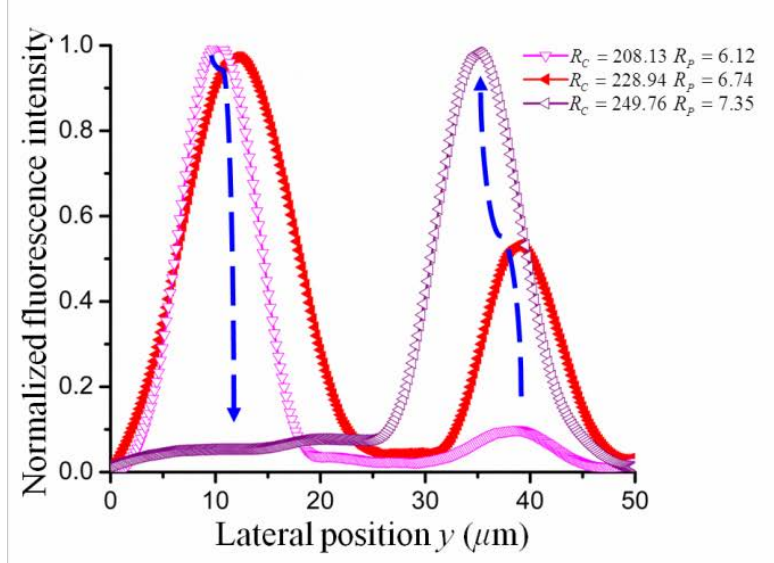

(c)

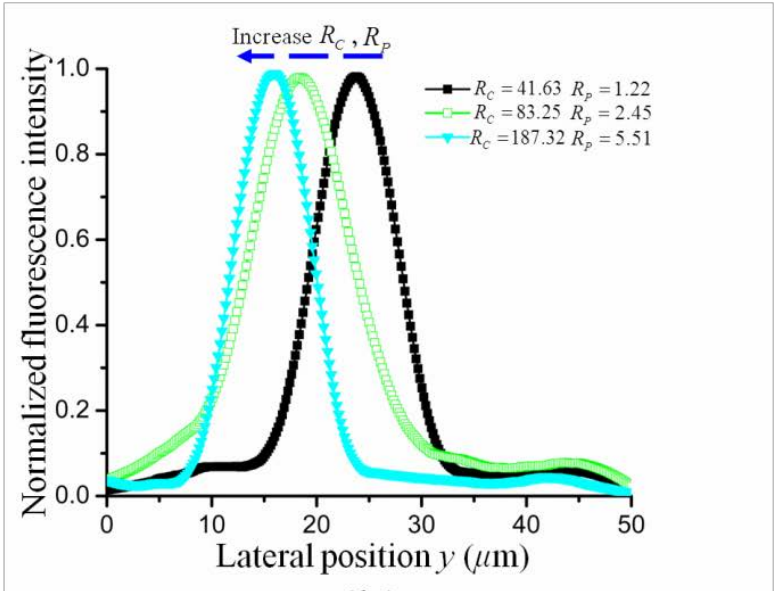

(b)

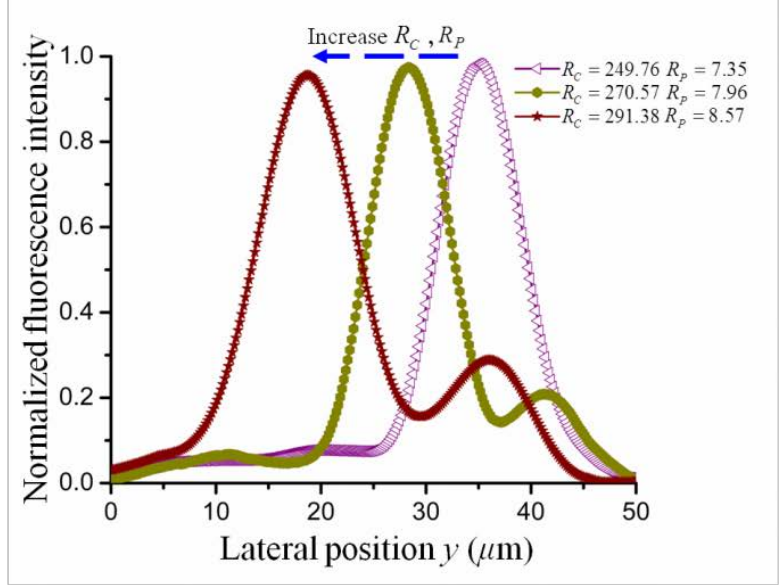

(d)

Figure 6. The normalized fluorescence intensity profile of $9.9 \mu \mathrm{m}$ particles along the channel width under different $R_{C}$ and $R_{P}$ at the outlet, representing the particles' distribution at the outlet. (a) The fluorescence profile at the outlet for three typical focusing patterns: single focusing streak on the cavity side, double focusing streaks on both side and single focusing streak on opposite side of cavity. (b) In the first focusing pattern, the focusing streak shifted to the cavity side when increasing flow rate (or $R_{C}$ ). (c) Transition procedures for three different focusing patterns. Continue to increase the flow rate at the end of first focusing pattern $\left(R_{C}=208.13, R_{P}=6.12\right)$, another focusing streak appeared on the cavity opposite side so that first focusing pattern transferred to second focusing pattern $\left(R_{C}=228.94, R_{P}=6.74\right)$. When $R_{C}$ reached 249.76 , the focusing streak on the cavity side disappeared, leaving only a focusing streak on opposite side. At this point, second focusing pattern transferred to 
third focusing pattern. (d) The focusing streak moved from right to left when increasing flow rate in third focusing pattern.

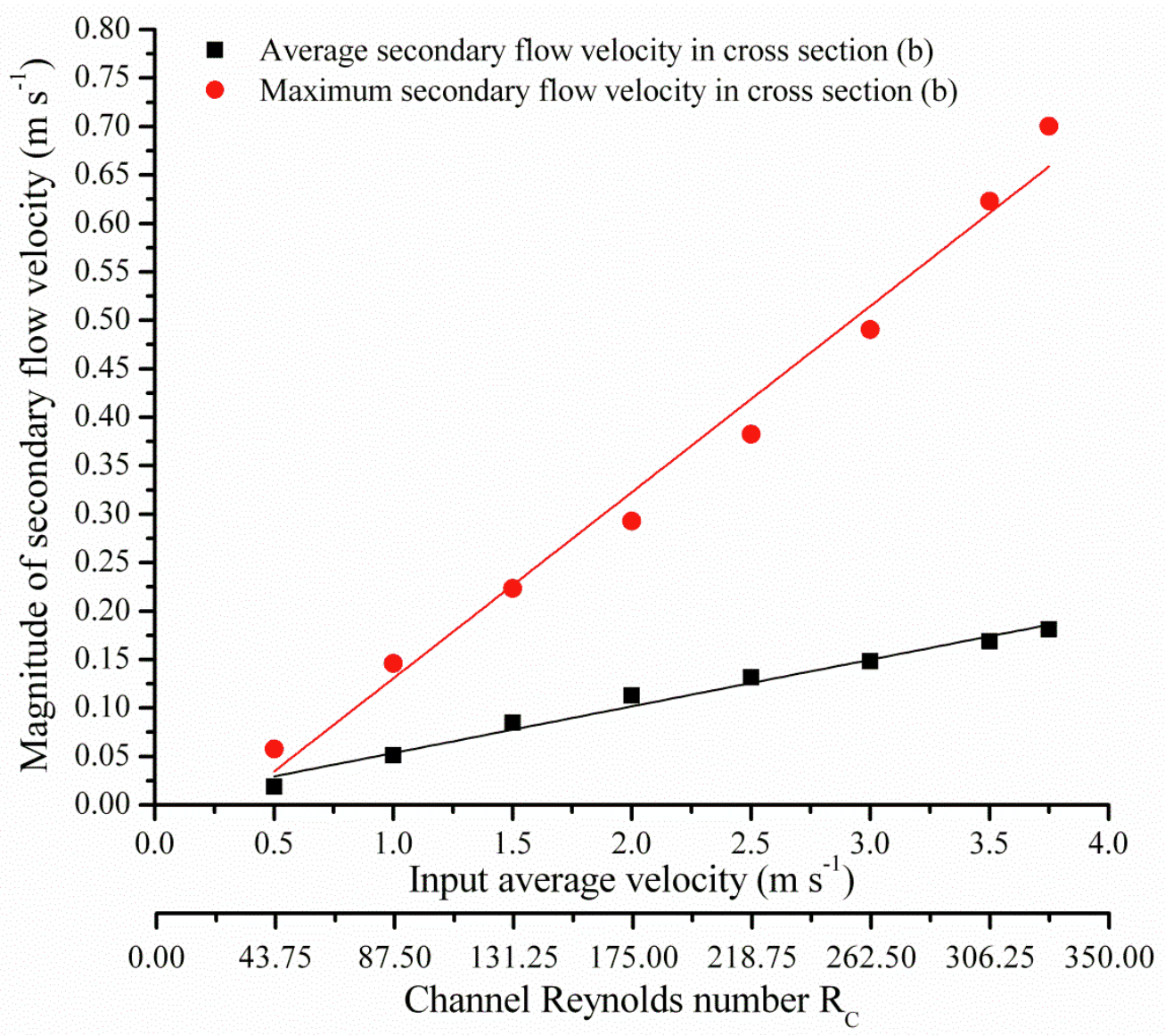

Figure 7. The magnitude of secondary flow is the average value of fluid lateral velocity in the cross-section, defined as $\oiint\left|v_{f}\right| d S$ / $S$. And the maximum magnitude of secondary flow is maximum absolute value of lateral fluid velocity in the cross section, defined as $\max _{S}\left(v_{f} \mid\right)$. The data are obtained from the flow field which is calculated by the numerical simulation. The square minimum method is used for fitting. 


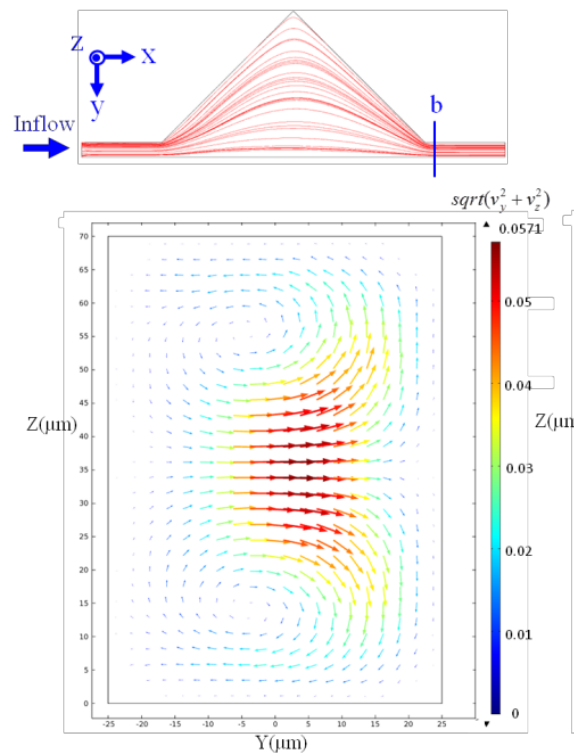

(a) $\mathrm{Rc}=41.63$
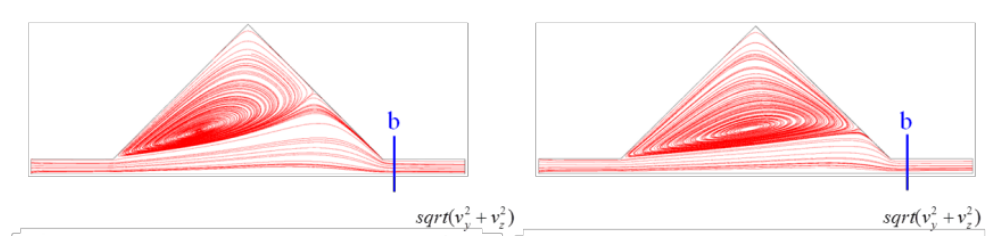

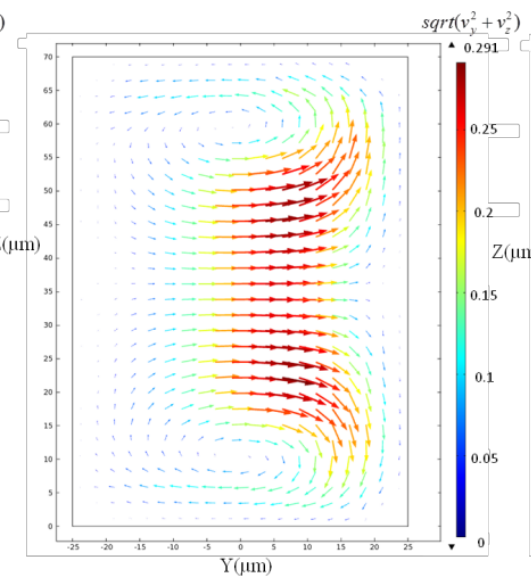

(b) $\mathrm{Rc}=166.5$

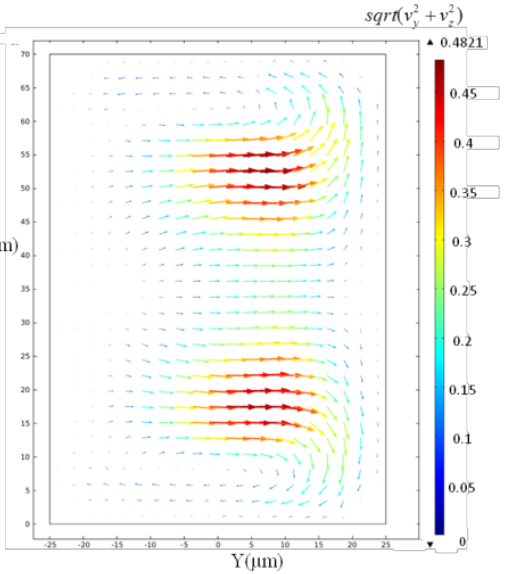

(c) $\mathrm{Rc}=249.76$

Figure 8. The Dean-like vortex under (a) $R_{C}=41.63$ : no horizontal vortex is formed, (b) $R_{C}=166.5$ : horizontal vortex is developed partially and (c) $R_{C}=249.76$ : horizontal vortex is developed completely. The cross section (b) is $925 \mu \mathrm{m}$ from the left beginning of the triangular cavity along $\mathrm{x}$ direction.

\subsection{Effects of particle size}

In order to study the effects of particle size on the focusing process, $4.8 \mu \mathrm{m}$ and $3.2 \mu \mathrm{m}$ particles suspensions were also introduced into the same microfluidic device. The fluorescence images and intensity profile of $4.8 \mu \mathrm{m}$ particles at the outlet were shown in figure 9 . Similar to the $9.9 \mu \mathrm{m}$ particles in the flow rate of $100 \mu \mathrm{l} \mathrm{min}^{-1}, 4.8 \mu \mathrm{m}$ particles were also focused to the central area of the channel $\left(R_{P}=0.28\right)$ (figure 9(i)). However, a focusing streak with $20 \mu$ m width in the centre of channel was observed for the $4.8 \mu \mathrm{m}$ particles (Figure 9(b)) and they began to expand into two sides symmetrically when $R_{P}$ exceeded 0.85 (figure 9a(ii) (v)), although single-particle focusing can be obtained with $9.9 \mu \mathrm{m}$ particles under this flow condition. This may be due to the much smaller inertial lift forces and secondary flow drag exerted on $4.8 \mu \mathrm{m}$ particles. So a much longer channel with more cavity arrays is expected to enable a single-particle focusing for these $4.8 \mu \mathrm{m}$ particles. 


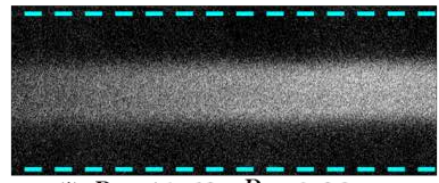

(i) $R_{C}=41.63, R_{P}=0.28$

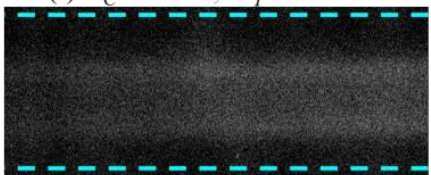

(ii) $R_{C}=124.88, R_{p}=0.85$

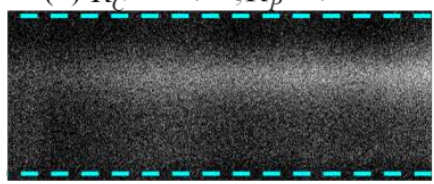

(iii) $R_{C}=166.50, R_{P}=1.12$

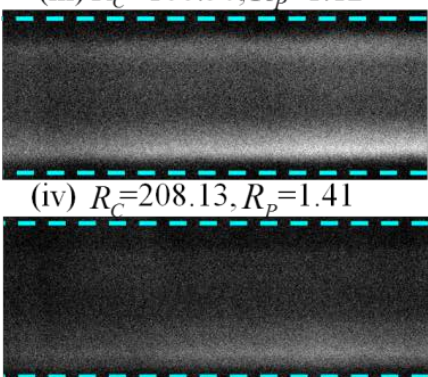

(v) $R_{C}=270.57, R_{P}=1.83$

(a)

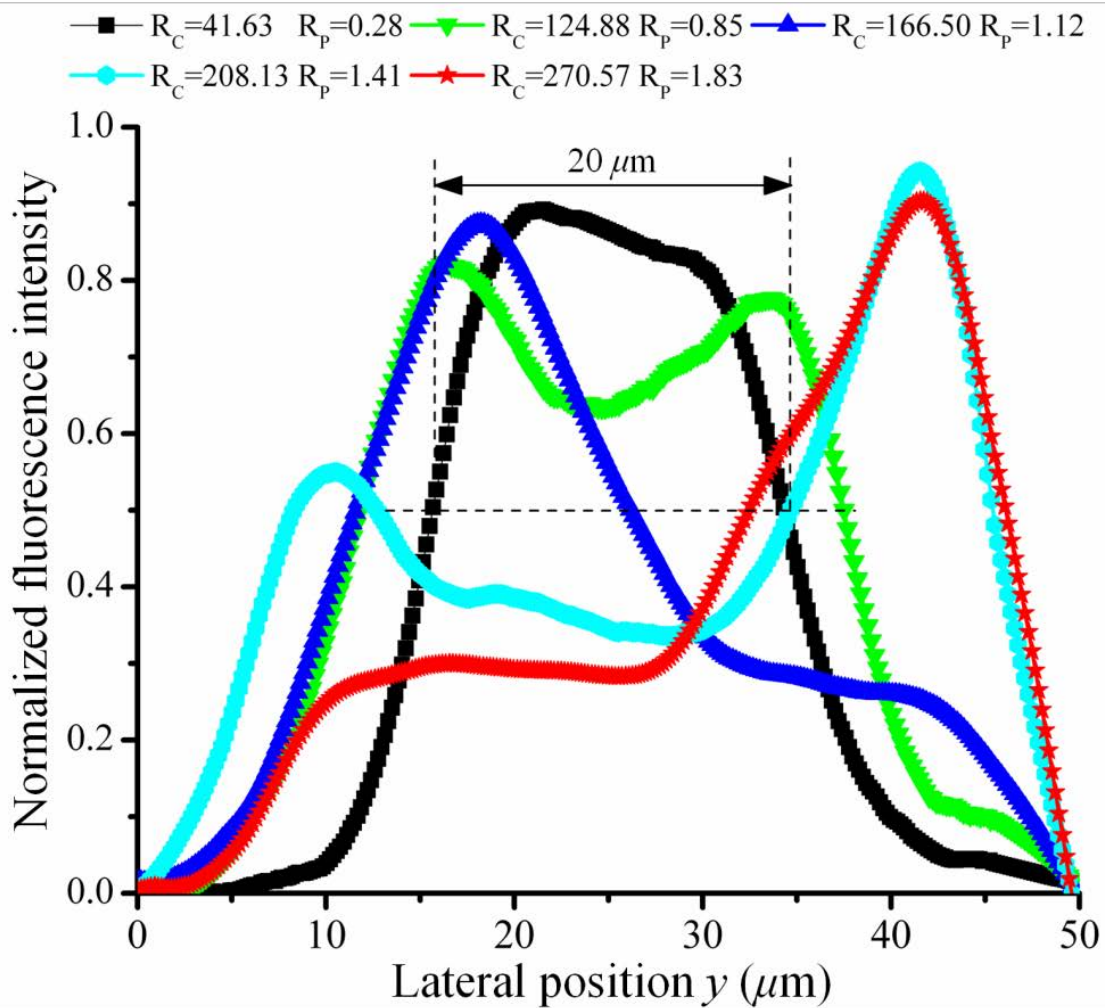

(b)

Figure 9. (a) Fluorescence images of $4.8 \mu \mathrm{m}$ particles at the outlet under different flow conditions. (b) Fluorescence intensity profile for $4.8 \mu \mathrm{m}$ particles at the outlet under the different $R_{C}$ and $R_{P}$.

$3.2 \mu$ m particles distributed almost uniformly at the outlet when inflow flow rate was $100 \mu \mathrm{lmin}{ }^{-}$ ${ }^{1}$, because particle Reynolds number was too small $\left(R_{P}=0.13\right)$, and the inertial effects were negligible (figure $10 \mathrm{a}(\mathrm{i})$ ). When the flow rate was increased to $400 \mu \mathrm{l} \mathrm{min}^{-1}\left(R_{P}=0.50\right)$, a focusing streak with 27 $\mu \mathrm{m}$ width in the centre of channel was also observed (figure 10a(ii)), which was similar with $4.8 \mu \mathrm{m}$ particles. When flow rate exceeded $500 \mu \mathrm{l} \mathrm{min}^{-1}\left(R_{P}=0.63\right)$, particles began to migrate and focus on both sides of channel although particle Reynolds number was still less than 1 (figure 10a(iii)). Additionally, the two focusing streaks got closer to channel walls by increasing the flow rate (figure 10a(iii) (v)), which agreed well with the inertial focusing phenomenon in a straight channel $[49,53]$. 


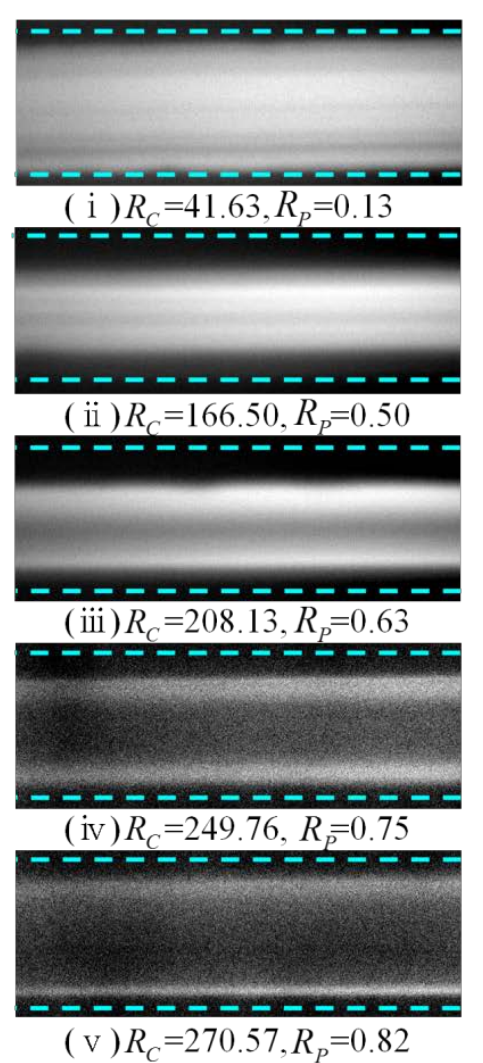

(a)

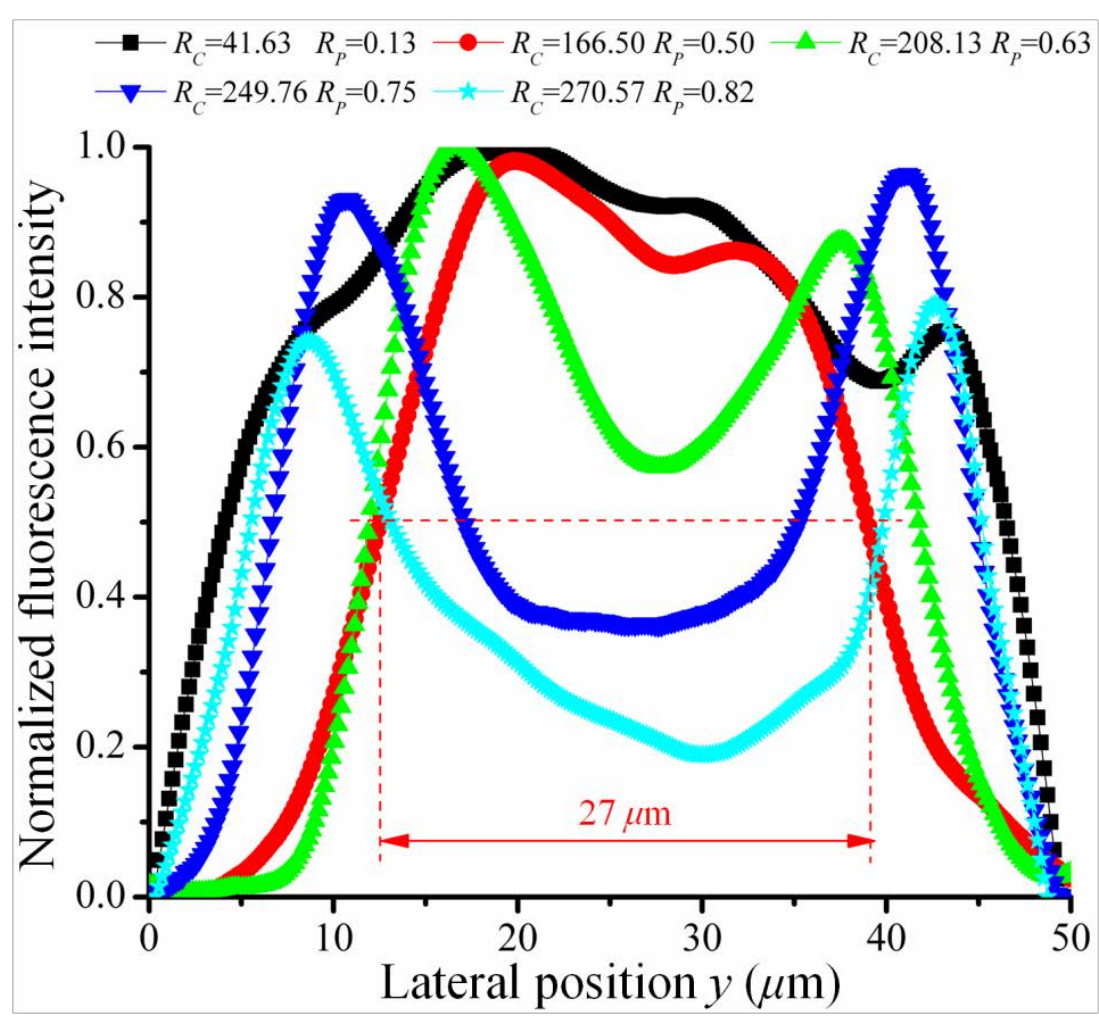

(b)

Figure 10. (a) Fluorescence images of $3.2 \mu \mathrm{m}$ particles at the outlet under different flow conditions. (b) Fluorescence intensity profile for $3.2 \mu \mathrm{m}$ particles at the outlet under the different $R_{C}$ and $R_{P}$.

\subsection{Effects of cavity geometry}

Besides the triangular cavity, cavity with other shapes, such as diamond and half circle, asymmetrically patterned on a straight channel were also applied to focus particles. These cavities' characteristic dimensions (diameter for half circular cavity, maximum diagonal line for diamond cavity) were the same with the triangular cavity as $900 \mu \mathrm{m}$. In these cavities, the expansion angle was equal to contraction angle. The $9.9 \mu \mathrm{m}$ particles suspension was introduced to get more obvious focusing phenomenon. By increasing flow rate from $50 \mu \mathrm{l} \mathrm{min}{ }^{-1}$, three different focusing patterns were also observed, as shown in figures 11 and 12. This phenomenon was similar to the abovereported triangular cavity arrays channel (triangular CEA channel). However, the flow conditions for each focusing pattern were quite different in different shaped CEA channels. For example, when flow rate was $400 \mu \mathrm{l} \mathrm{min}^{-1}\left(R_{C}=166.50, R_{P}=4.90\right)$, the first focusing pattern existed in triangular CEA channel (figure 3(a)), whereas second focusing pattern and third focusing pattern were already happened in half circular and diamond CEA channels, respectively (figures 11(b) and 12(c)). It indicates that the focusing pattern and its working condition can be adjusted by the geometry of cavity. The working conditions for three different focusing patterns in CEA channel with different expansion and contraction angles are plotted in figure 13 . We can see that the trigger channel 
Reynolds number (the very start flow condition for each focusing pattern) for the second and third focusing patterns decreases with increasing cavity's expansion and contraction angle. So when first focusing pattern is applied to flow cytometry, a smaller expansion and contraction angle is preferred in order to have a wider available working area. However, to use the third focusing pattern for the particles trapping and circulation, a larger expansion and contraction angle is favoured because vortex can be generated in a much lower flow rate, which will significantly reduce the possibility of microfluidic chip failures due to the inadequate bonding and sealing strength.

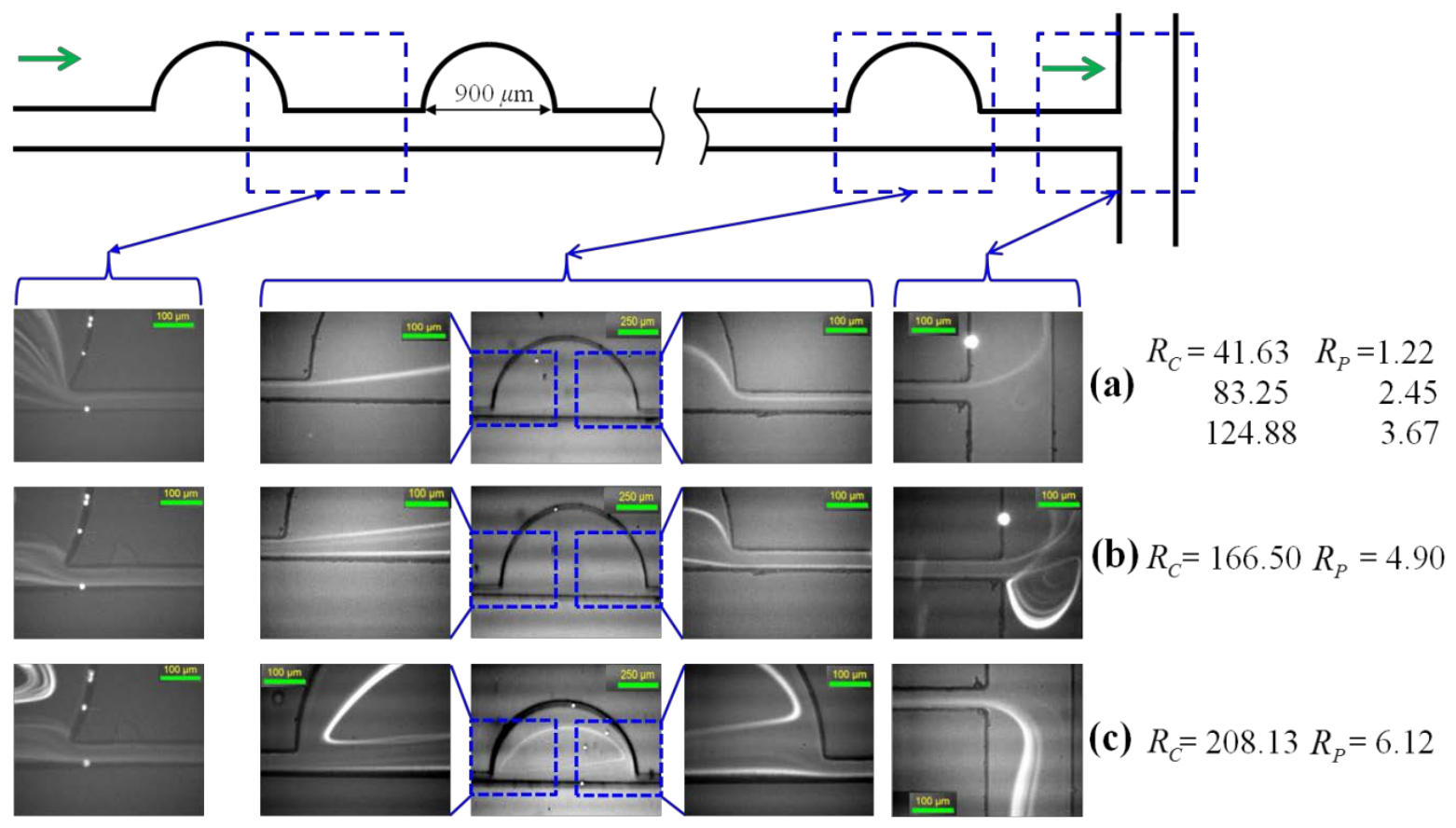

Figure 11. Three different focusing patterns in a straight channel with asymmetrically patterned half circular cavity arrays (particles diameter $=9.9 \mu \mathrm{m}$ ). 


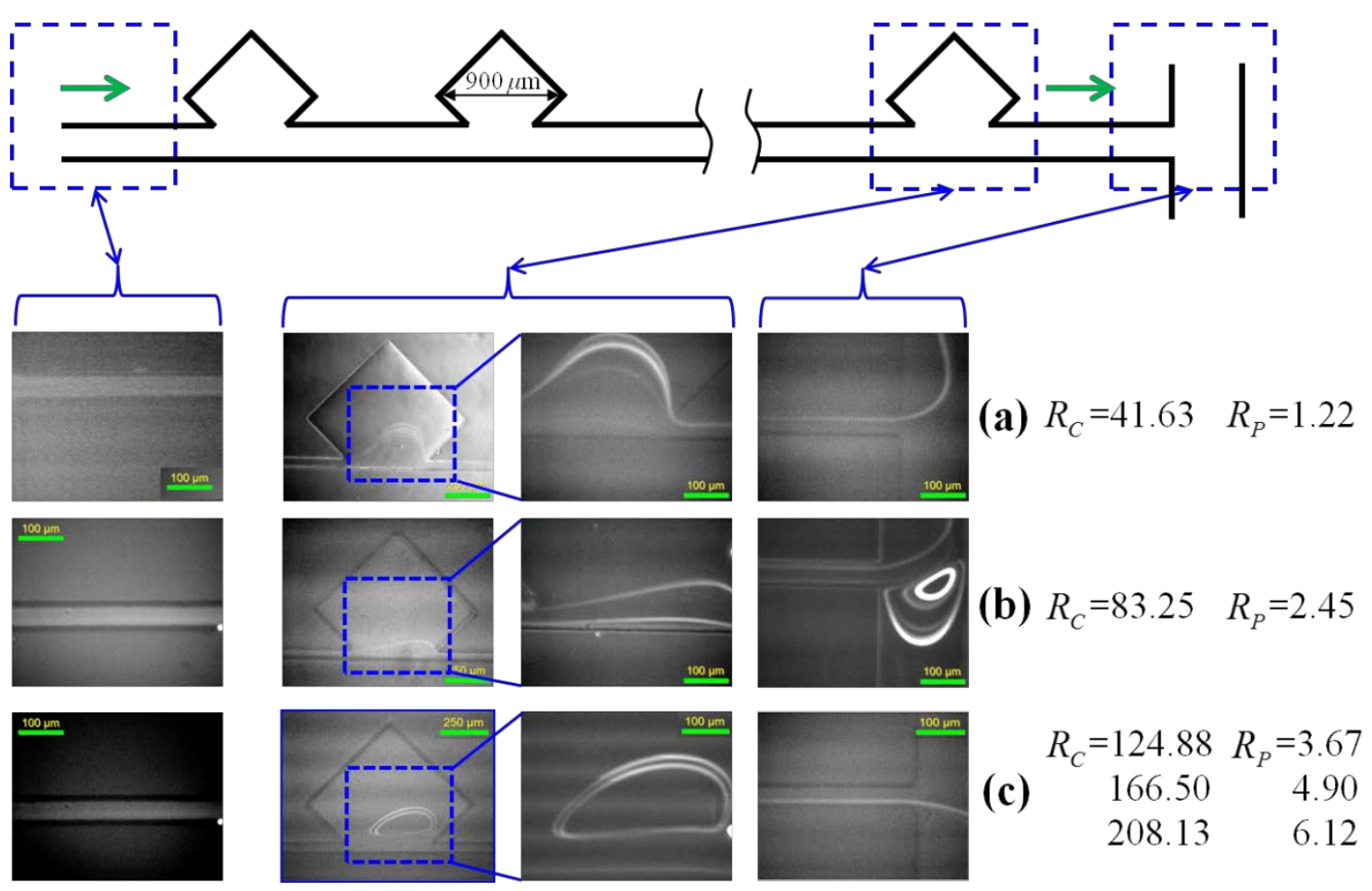

Figure 12. Three different focusing patterns in a straight channel with asymmetrically patterned diamond cavity arrays (particles diameter $=9.9 \mu \mathrm{m}$ ).

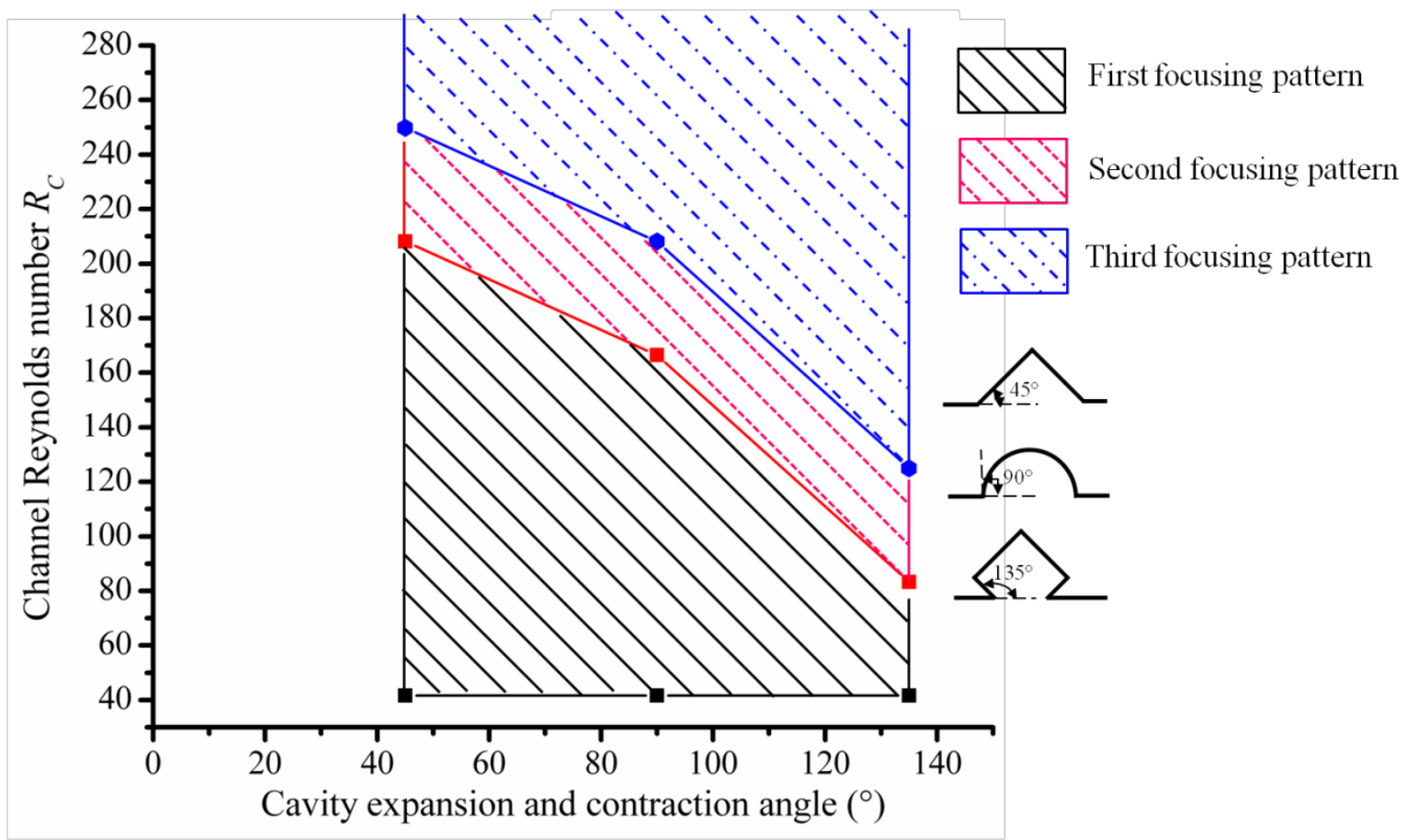

Figure 13. The working conditions for three different focusing patterns in cavity arrays channel with different expansion and contraction angles: $45^{\circ}$ (triangular cavity), $90^{\circ}$ (half circular cavity), $135^{\circ}$ (diamond cavity).

\section{Conclusion}


A novel inertial microfluidic device with asymmetrically patterned triangular cavity arrays to effectively focus particles was presented. Two secondary flows in cross-sectional and horizontal planes were innovatively employed together to modify inertial focusing in a simple single-layer microfluidic chip. Three different focusing patterns were observed, and their focusing mechanism was discussed up to $R_{P}=7.35$. The first focusing pattern is suggested for the application of flow cytometry, where detection unit can be placed in a specific lateral position of downstream according to the working flow condition. The second focusing pattern can be used to filter particles (such as blood cells for the extraction of blood plasma). Outlets can be added on the two sides of main channel, to extract focused particles from mainstream. The third focusing pattern can meet the demands where both particles focusing and trapping are needed at the same time. The proposed focusing method has following advantages: (i) sheathless, no sheath flow is used to eliminate the risk of dilution and contamination; (ii) high throughput, the flow rate can be as high as $700 \mu \mathrm{lmin}^{-1}$ in a single channel; (iii) good focusing performance (especially for large particles), a single-particle focusing can be achieved for $9.9 \mu \mathrm{m}$ particles, which reaches the performance of most reported hydrodynamic microfluidic chip [11, 35, 44, 48, 54]; (iv) flexible focusing ability, three different focusing patterns can satisfy various demands, with their working condition adjustable by the geometry of cavity. Overall, this inertial microfluidic device can provide a continuous, sheathless and high-throughput focusing performance, which can be potentially applied to high-speed on-chip flow cytometry or filtration of bio-particles.

\section{Acknowledgement}

This work is partially supported by the University of Wollongong through a UIC grant. The authors thank Professor Dino Di Carlo, University of California, Los Angeles for the useful discussions.

\section{References}

[1] Di Carlo D, Jon F, Irimia D, Tompkins RG and Toner M 2008 Anal. Chem. 80 2204-11.

[2] Goda K, Ayazi A, Gossett DR, Sadasivam J, Lonappan CK, Sollier E, et al. 2012 Proc. Natl. Acad. Sci. U.S.A. 109 11630-5.

[3] Holmes D, She JK, Roach PL and Morgan H 2007 Lab Chip 7 1048-56.

[4] Hemmatifar A, Saidi MS, Sadeghi A and Sani M 2012 Microfluid. Nanofluid. 1-12.

[5] Li M, Li S, Cao W, Li W, Wen W and Alici G 2012 J. Micromech. Microeng. 22095001.

[6] Liang L and Xuan X 2012 Microfluid. Nanofluid. 1-7.

[7] Zeng J, Chen C, Vedantam P, Brown V, Tzeng T-RJ and Xuan X 2012 J. Micromech. Microeng. 22105018.

[8] Shi J, Mao X, Ahmed D and Huang TJ 2008 Lab Chip 8 221-3.

[9] Wang Z and Zhe J 2011 Lab Chip 11 1280-5.

[10] Di Carlo D 2009 Lab Chip 9 3038-46.

[11] Di Carlo D, Irimia D, Tompkins RG and Toner M 2007 Proc. Natl. Acad. Sci. U.S.A. 104 18892.

[12] Cherukat P and Mclaughlin JB 1994 J. Fluid Mech. 263 1-18.

[13] Saffman PG 1965 J. Fluid Mech. 22 385-400.

[14] Drew DA 1978 J. Fluid Mech. 88 393-400. 
[15] Segre G 1961 Nature 189 209-10.

[16] Segre G and Silberberg A 1962 J. Fluid Mech. 14 136-57.

[17] McLaughlin JB 1993 J. Fluid Mech. 246 249-65.

[18] JOSEPH DD and OCANDO D 2002 J. Fluid Mech. 454 263-86.

[19] ASMOLOV ES 1999 J. Fluid Mech. 381 63-87.

[20] Chun B and Ladd A 2006 Phys. Fluids 18031704.

[21] Tanaka T, Ishikawa T, Numayama-Tsuruta K, Imai Y, Ueno H, Yoshimoto T, et al. 2012 Biomed. Microdevices 1-9.

[22] Bhagat AAS, Kuntaegowdanahalli SS and Papautsky I 2008 Phys. Fluids 20101702.

[23] Mach AJ and Di Carlo D 2010 Biotechnol. Bioeng. 107 302-11.

[24] Humphry KJ, Kulkarni PM, Weitz DA, Morris JF and Stone HA 2010 Phys. Fluids 22 081703.

[25] Papautsky I and Bhagat AAS 2012 Spiral microchannel particle separators, straight microchannel particle separators, and continuous particle separator and detector systems. Google Patents.

[26] Hou HW, Gan HY, Bhagat AAS, Li LD, Lim CT and Han J 2012 Biomicrofluid 6024115.

[27] Seo J, Lean MH and Kole A 2007 J. Chromatogr. A 1162 126-31.

[28] Seo J, Lean MH and Kole A 2007 Appl. Phys. Lett. 91 033901--3.

[29] Kuntaegowdanahalli SS, Bhagat AAS, Kumar G and Papautsky I 2009 Lab Chip 9 2973-80.

[30] Vermes I, Weitz DA and van den Berg A 2012 Lab Chip.

[31] Bhagat AAS, Kuntaegowdanahalli SS and Papautsky I 2008 Lab Chip 8 1906-14.

[32] Yoon DH, Ha JB, Bahk YK, Arakawa T, Shoji S and Go JS 2008 Lab Chip 9 87-90.

[33] Gossett DR and Carlo DD 2009 Anal. Chem. 81 8459-65.

[34] Oozeki N, Ookawara S, Ogawa K, Löb P and Hessel V 2009 AIChE J. 55 24-34.

[35] Oakey J, Applegate Jr RW, Arellano E, Carlo DD, Graves SW and Toner M 2010 Anal. Chem. 82 3862-7.

[36] Lee MG, Choi S, Kim HJ, Lim HK, Kim JH, Huh N, et al. 2011 Appl. Phys. Lett. $98253702--$ 3.

[37] Lee MG, Choi S and Park JK 2011 J. Chromatogr. A 1218 4138-43.

[38] Lee MG, Choi S and Park JK 2010 Biomed. Microdevices 12 1019-26.

[39] Hur SC, Mach AJ and Di Carlo D 2011 Biomicrofluid 5022206.

[40] Mach AJ, Kim JH, Arshi A, Hur SC and Di Carlo D 2011 Lab Chip 11 2827-34.

[41] Shelby JP and Chiu DT 2004 Lab Chip 4 168-70.

[42] Chiu DT 2007 Anal. Bioanal. Chem. 387 17-20.

[43] Shelby JP, Lim DSW, Kuo JS and Chiu DT 2003 Nature 425 38-.

[44] Park JS, Song SH and Jung HI 2009 Lab Chip 9 939-48.

[45] Lee MG, Choi S and Park JK 2009 Lab Chip 9 3155-60.

[46] Bhagat AAS, Kuntaegowdanahalli SS and Papautsky I 2009 Microfluid. Nanofluid. 7 217-26.

[47] Stroock AD, Dertinger SKW, Ajdari A, Mezić I, Stone HA and Whitesides GM 2002 Science 295647.

[48] Chung AJ, Gossett DR and Di Carlo D 2012 Small.

[49] Di Carlo D, Edd JF, Humphry KJ, Stone HA and Toner M 2009 Phys. Rev. Lett. 10294503.

[50] Sharp KV and Adrian RJ 2004 Exp. Fluids 36 741-7.

[51] Martel JM and Toner M 2012 Phys. Fluids 24032001.

[52] Zhou J and Papautsky I 2013 Lab Chip.

[53] MATAS J-P, MORRIS JF and GUAZZELLI É 2009 J. Fluid Mech. 621 59-67.

[54] Choi S, Song S, Choi C and Park JK 2008 Small 4 634-41. 